\title{
Erratum zu: Eine Verschärfung des \\ Unvollständigkeitssatzes der Zahlentheorie \\ E. Specker
}

Erratum zu: E. Specker, Ein Verschärfung des Unvollständigkeitssatzes der Zahlentheorie, DOI 10.1007/978-3-0348-9259-9_11

Der Name des Autors wurde in Kapitel 11 dieses Buches falsch erfasst. E. Speckek sollte als E. Specker gelesen werden. Dies wurde korrigiert. 